\title{
Genetic Diversity of Osmophilic Yeasts Isolated from Indonesian Foods with High Concentration of Sugar
}

\author{
RIDAWATI $^{1 *}$, BETTY SRI LAKSMI JENIE ${ }^{1}$, ITA DJUWITA², AND WELLYZAR SJAMSURIDZAL \\ ${ }^{\prime}$ Department of Food Science and Technology, Faculty of Agricultural Technology, Institut Pertanian Bogor, \\ Darmaga Campus, Bogor 16680, Indonesia; ${ }^{2}$ Department of Anatomy Physiology and Pharmacology, \\ Faculty of Veterinary Medicine, Institut Pertanian Bogor, Darmaga Campus, Bogor 16680, Indonesia \\ ${ }^{3}$ Department of Biology, Faculty of Mathematics and Natural Sciences, Universitas Indonesia,
} UI Campus, Depok 16424, Indonesia

\begin{abstract}
Isolation of osmophilic yeasts from a total of 70 samples consisting of jam, sweet condensed milk, honey, sweet soy sauce, and palm sugar was conducted. Sixty-eight osmophilic yeasts were isolated from strawberry jam, pineapple jam, and honey from South Sumatera. No yeast was obtained from condensed milk, honey from Sumbawa, sweet soy sauce, and palm sugar. Sequence analysis based on the ITS region showed that isolates were identified as five species belong to two genera, Candida and Sterigmatomyces. Those isolates were distributed in 5 species, C. metapsilosis, C. etchellsii, C. parapsilosis, C. orthopsilosis, and S. halophilus. C. etchellsii was the predominant species in South Sumatera honey, while C. parapsilosis group was predominant species in jams. Those species were reported as osmophilic yeasts. In both jams and honey we found $C$. parapsilosis and C. metapsilosis, whilst $C$. orthopsilosis was found only in pineapple jam. Phylogenetic analysis based on sequence of ITS region showed that most of the osmophilic yeasts (67 out of 68 isolates) were located in the phylum Ascomycota and only one isolate Sterigmatomyces halophilus NN38 from pineapple jam was located in the phylum Basidiomycota.
\end{abstract}

Key words: genetic diversity, osmophilic yeasts, food with high sugar concentration

Spoilage of food products by microorganisms results in the waste of valuable resources and causes large financial losses for the food industry that will inevitably affect the consumer. High sugar products are targets for spoilage by yeast. Various kinds of traditional high sugar foods in Indonesia such as jam, sweet condensed milk, sweet soy sauce and honey have the potential to be spoiled by yeast. Some yeasts have the ability to grow or cause spoilage in foods, especially products with low $\mathrm{pH}$, generally 5.5 or lower, and other products by the presence of sugars as carbon sources. The adverse media inflicted by the low $\mathrm{pH}$, low oxygen levels, and high sugar concentration of these products prevents the growth of most organisms. However, these hurdles do not inhibit the growth of many spoilage yeasts, especially osmophilic yeasts.

The reported food and beverage spoilage yeasts include a relatively large number of species representing both ascomycetes and basidiomycetes as has been published. Many yeasts were frequently described as being osmophilic, suggesting a habitat restricted to a high solute (in this case, sugar) environment. The $\mathrm{a}_{\mathrm{w}}$ of typical concentrated syrups (more than 50\% soluble solids) ranges from 0.82-0.94. Depending upon the solute and its concentration (salt, sugar, or glycerol), some yeasts are able to grow at relatively low temperatures. Many osmophilic yeasts had been identified as spoilage agents in fruit concentrates and juices (Arias et al. 2002; Fitzgerald et al. 2004) and syrup (Ancasi et al. 2006).

Osmophilic yeasts can create a spoilage problem in food storage, because they are able to grow under conditions of high salt or sugar content. Aside from their ability to grow in the restrictive habitat of high osmotic pressure, many yeasts

\footnotetext{
*Corresponding author and recent address, Department of Food and Nutrition, Faculty of Engineering, Universitas Negeri Jakarta, Jalan Rawamangun Muka, Jakarta 13220, Indonesia; Phone: +62-21-4715094; Fax: +62-21-4715094; Email: ridawatis@yahoo.co.id
}

are also extraordinarily resistant to common preservatives such as sulphur dioxide, sorbic acid, benzoic acid and acetic acid (Warth 1985). Yeasts can become adapted to preservatives by preexposure to low levels of these materials.

Many studies regarding these microorganisms have been carried out and indicate a desire to understand the diversity of yeasts in high sugar foods to prevent food spoilage. Morphologically and physiologically, these osmophilic yeasts were found to be extremely similar to one another, with species differentiation being based mainly on the high sugar content of media and the ability to form ascospores or pseudomycelia. In order to understand the species interrelationships of these yeasts better, a phylogenetic study based on ITS sequence of $18 \mathrm{~S}$ rDNA was carried out upon all strains isolated in this work. It is important to understand the characteristics of the isolated yeasts. Molecular comparisons have provided an understanding of yeast phylogeny that was not possible from analyses of morphology and physiology.

Gene-sequence determinations have also provided a rapid, accurate means for identification of individual strains to the species level. Sequencing of species-diagnostic genes represents the most accurate means for isolate identification, and several rapid identification methods using molecular probes based on these sequences have been developed and are becoming available to food microbiology laboratories. Over the last decade, different polymerase chain reaction (PCR) techniques and nuclear sequence analysis have been used for identifying many yeasts for the study of phylogeny. The ITS region of ribosomal DNA had been used for this purpose. The use of 5.8S-ITS sequence is the best tool for rapid and accurate identification of yeast. Some studies have reported that the ITS1 and ITS2 regions had higher degrees of variability and therefore higher differentiating power than D1/D2 region for closely related species (Tavanti et al. 2005; 
Kocsube et al. 2007). Partial sequencing of ITS region is convenient for the rapid characterization of yeast. Using sequences available in the GenBank database, sequences obtained could be used to compare the similarities of gene fragments among the currently recognized yeast species.

In view of these issues, the objectives of the present study were (i) to obtain isolates of osmophilic yeasts from high sugar food products, (ii) to identify their genetic diversity and to estimate the taxonomic boundaries of the osmophilic species using the ITS rDNA sequence information, and (iii) to reveal the phylogenetic relationship between osmophilic yeast isolated from many kinds of foods with high concentration of sugar in Indonesia.

\section{MATERIALS AND METHODS}

Isolation of Osmophilic Yeasts. A total of 70 samples of local high sugar food products (jams, sweet condensed milk, honey, sweet soy sauce, and palm sugar) were collected from different markets in Jakarta, Bogor, Sumbawa, and South Sumatera. Osmophilic yeasts were isolated from these samples. A procedure for isolation and preparation of osmophilic yeast was the use of potato dextrose broth (PDB) and potato dextrose agar (PDA) (Oxoid) enriched with various concentrations $(10-60 \% \mathrm{w} / \mathrm{v})$ of sucrose were conducted. Subsequently, the colonies which formed were isolated and purification by the quadrant streak method.

DNA Extraction and PCR Analysis. Total DNA was extracted from osmophilic yeasts by boiling method according to Sjamsuridzal and Oetari (2003). The DNA extract was amplified by PCR technique using ITS4 and ITS5 primers (White et al. 1990). PCR reactions were prepared in $25 \mu \mathrm{L}$ total volume containing $9 \mu \mathrm{L}$ DNA template; 0.5 pmol of each ITS4 and ITS5 primer; PCR

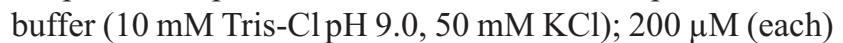
dATP, dGTP, dCTP, and dTTP; $1.5 \mathrm{mM} \mathrm{MgCl}$; 2.5 unit pureTaq DNA polymerase (GE Healthcare Kit). The amplification procedure of the rDNA ITS region was carried out using a published method (White et al. 1990). The amplification profile consisted of 40 cycles of denaturation at $95^{\circ} \mathrm{C}$ for $15 \mathrm{sec}$, primer annealing at $58^{\circ} \mathrm{C}$ for $30 \mathrm{sec}$, and primer extension at $68^{\circ} \mathrm{C}$ for $1 \mathrm{~min}$; followed by post extension at $68^{\circ} \mathrm{C}$ for $10 \mathrm{~min}$. PCR products were analyzed on $2 \%(\mathrm{w} / \mathrm{v})$ agarose gel, stained with ethidium bromide, and visualized under UV light using the Chemidoc Gel System (Biorad).

Partial Sequencing of ITS Region and Phylogenetic Analysis. DNA for sequencing was amplified using a 9600 Perkin-Elmer Cetus Thermal Cycler apparatus as described by White et al. (1990) with reverse primer (ITS5). DNA sequencing was performed on ABI Prism 310 DNA Sequencer with protocols supplied by the manufacturer. The sequencing results were compared with DNA sequences from GenBank database at National Center of Biotechnology Information (NCBI) using the Basic Local Alignment Search Tool (BLAST) program for screening sequence similarity (Altschul et al. 1997). Sequence alignments were performed by Clustal X1.83 program.
The phylogenetic tree was constructed using the neighborjoining (NJ) method (Saitou and Nei 1987).

\section{RESULTS}

Isolation of Osmophilic Yeasts. The isolates could not grow in media supplemented with 10,20 , and $30 \%(\mathrm{w} / \mathrm{v})$ sucrose, whereas most of the isolates showed a higher cell number at $50 \%(\mathrm{w} / \mathrm{v})$ sucrose, and some growth was found in the presence of $40 \%(\mathrm{w} / \mathrm{v})$ sucrose and even in the presence of $60 \%(\mathrm{w} / \mathrm{v})$ sucrose. The result showed that osmophilic yeast strains grew better on PDA enriched with $50 \%(\mathrm{w} / \mathrm{v})$ sucrose than PDA alone. Colony size of the yeast grown in the media with lower concentration of sugar were smaller than in the media with a high sugar concentration (results not shown).

A feature of the yeast strains isolated from the high sugar foods were ascomycetous characteristics. These yeasts are osmophilic and could frequently grow under extreme conditions. Sixty eight osmophilic yeasts could be isolated from strawberry jam $\left(\mathrm{a}_{\mathrm{w}} 0.84\right)$, pineapple jam $\left(\mathrm{a}_{\mathrm{w}} 0.84\right)$, and honey from South Sumatera $\left(a_{w} 0.72\right)$. Colonies were white to tannish white, creamy, shiny, smooth and produced a faintly acidic aromatic odor. No yeast was obtained from sweet condensed milk $\left(a_{w} 0.84\right)$, sweet soy sauce $\left(a_{w} 0.68\right)$, honey from Sumbawa $\left(\mathrm{a}_{\mathrm{w}} 0.67\right)$, and palm sugar samples $\left(\mathrm{a}_{\mathrm{w}}\right.$ 0.63) (data not shown).

Analysis of the ITS Regions and Phylogenetic Analysis. Amplification of the ITS1-5.8S-ITS2 regions from the sixty eight osmophilic yeasts generated PCR products ranging in size from 400 to $500 \mathrm{bp}$. The profiles of bands representing the osmophilic yeasts were shown in Fig 1. One isolate (NN38) appeared to have a different profile from the others, so this isolate may be a different species. Phylogenetic trees of rDNA sequences were constructed based on the distance matrix methods as stated in the methodology. Sixty-seven of the sequences clustered in the phylum Ascomycota, however one isolate formed a different branch far away from the others and clustered within the phylum Basidiomycota (Fig 2). Thirteen yeast strains, representing four Basidiomycota-lineage and nine Ascomycota-lineage and their accession numbers obtained from GenBank are listed in Table 1.

Comparative analysis of the ITS region data revealed that the fifty-two strains of Candida parapsilosis group were highly related to one another, exhibiting sequence identity values that ranged from 99 to $100 \%$ (data not shown). Sequence analysis based on ITS region showed that 68 isolates were identified as five species belonging to two genera Candida and Sterigmatomyces. Those isolates were distributed in 5 species, i.e. C. metapsilosis (23), C. etchelsii (15), C. parapsilosis (25), C. orthopsilosis (4), and $S$. halophilus (1). C. etchellsii was the predominant species in South Sumatera honey and the $C$. parapsilosis group was the predominant species in strawberry jam and pineapple jam. In both jams and honey, $C$. parapsilosis and $C$. metapsilosis were found, while C. orthopsilosis was found only in pineapple jam (data not shown). 


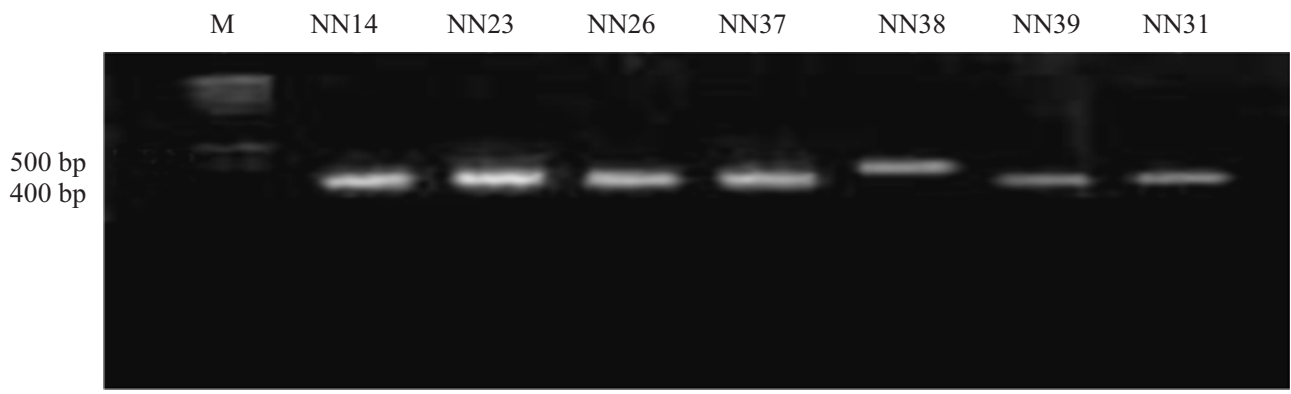

Fig 1 Gel electrophoresis images showing PCR products of yeast ITS region rDNA (about 400-500 bp in size). M, Marker; NN14, NN23, NN26, NN27, $\mathrm{NN} 38, \mathrm{NN} 39, \mathrm{NN} 31$, isolate from pineapple jam.

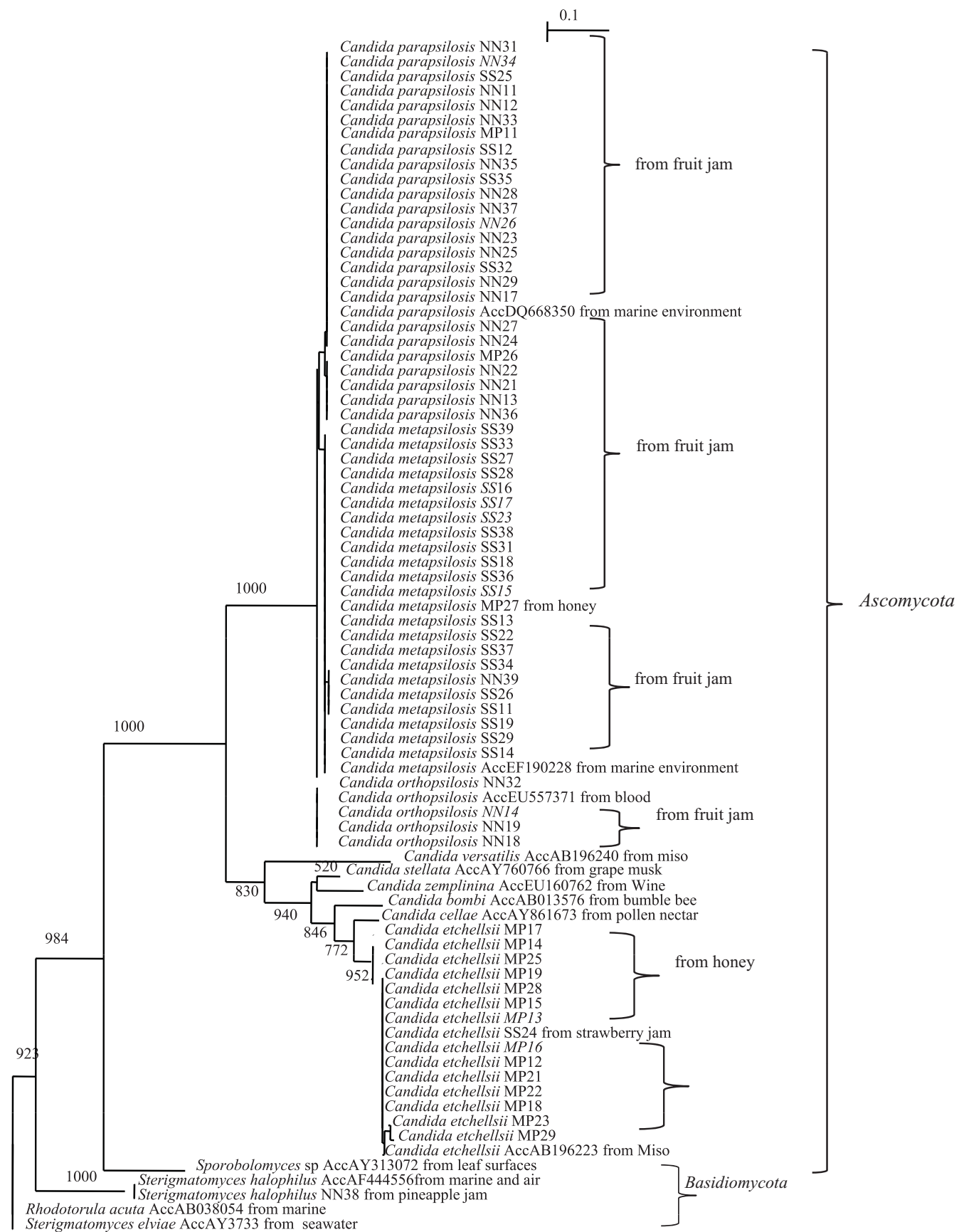

Fig 2 Dendrogram showing the phylogenetic relationship of Candida parapsilosis and C. etchellsii isolates from jam and honey with related yeast species based on ITS region. Bootstrap values, expressed as percentages of 1000 replications, are given at branch points (only values $>50 \%$ are shown). 
Table 1 Species name, source, and GenBank accession numbers of yeasts included in the phylogenetic analysis

\begin{tabular}{lll}
\hline Species name & Accesion number & Source \\
\hline Candida versatillis & AB196240 & Miso* \\
C. zemplinina & AY160762 & Wine \\
C. stellata & AY160766 & Grape musk \\
C. bombi & AB013576 & Bumblebee \\
C. cellae & AY861673 & Pollen nectar \\
C. etchellsii & AB196223 & Miso* \\
C. parapsilosis & DQ668350 & Marine \\
& & environment \\
C. metapsilosis & EF190228 & Marine \\
& & environment \\
C. orthopsilosis & EU557371 & Blood sample \\
Sporobolomyces sp. & AY313072 & Leaf surfaces \\
Rhodotorula acuta & AB038054 & Marine \\
Sterigmatomyces elviae & AY373390 & Seawater \\
S. halophilus & AF444556 & Marine and air \\
\end{tabular}

* A thick fermented paste made of cooked soybeans, salt, and often rice or barley, and used especially in making soups and sauces.

\section{DISCUSSION}

Osmophilic yeasts have been isolated from Indonesian foods with high sugar concentration sugar and water activity between $0.72-0.84$. Typically, the shelf-life of these products could be extended by pasteurization, evaporation, and/or the addition of chemical preservatives such as acid or benzoic. However, these preservation methods do not inhibit the growth of many spoilage-causing yeasts, especially osmophilic yeasts. A total of 68 isolates could be obtained from various samples. The results showed that osmophilic yeast strains grew better on PDA enriched with $50 \%(\mathrm{w} / \mathrm{v})$ sucrose than PDA alone.

Water activity refers to the availability of water in foods or beverages and represents the amount of water that was available for microorganisms growth. A majority of food spoilage yeasts had been isolated from food with low water activity (foods with $65 \%$ (w/v) sucrose or $15 \%$ (w/v) $\mathrm{NaCl}$ or foods with $\mathrm{a}_{\mathrm{w}}$ 0.87) and could survive on $\mathrm{a}_{\mathrm{w}} 0.80-0.85$ as reported by Beuchat (1983). In most cases, yeast contamination in these products occurred on the farm and they were therefore present in the fresh products.

No yeast was obtained from sweet condensed milk (SCM), even though these samples had a water activity value of 0.84 . Aside of water activity, the main factor causing a lack of yeast obtained from SCM was the processing of these products. In SCM manufacturing, heat-treated milk containing sugar is taken to the evaporator, where it is concentrated by heating the mixture $93.58^{\circ} \mathrm{C}$ for $3 \mathrm{~min}$ depending on the desired outcome of viscosity, bacteriological quality, and other physico-chemical properties, then evaporated, cooled, and tinned or bottled under hygienic conditions (Beuchat 1983; Ali and Randal 2002). The method for preserving SCM using aseptic technology means contaminant osmophilic yeast could not survive in these products.
Sweet soy sauce $\left(a_{w} 0.68\right)$, honey from Sumbawa $\left(a_{w}\right.$ $0.67)$ and palm sugar $\left(a_{w} 0.63\right)$ have low water activity. No yeasts were obtained from these products. Some unique foods like sweet soy sauce appear to be a high moisture product, but because salt, sugars, or other ingredients bind the water content, their water activities become quite low. The presence of sugar in such a high concentration will inhibit the growth of yeast contaminants.

From sequencing results, a total of 68 strains-sequencedata were aligned together with known sequences of several species from the GenBank database. A phylogenetic tree was constructed using the NJ method as stated in the methodology, using S. halophilus as an outgroup. All species of C. parapsilosis, C. orthopsilosis, and C. metapsilosis were closely related and formed a single cluster (Fig 2). The species of this cluster were isolated from various sources, including honey, strawberry jam, and pineapple jam. They are widely distributed in nature and are common spoilage yeast in the food industry. Tavanti et al. (2005) and Andrew et al. (2009) had reported that all isolates within each group (C. parapsilosis, C. metapsilosis, C. orthopsilosis) had similar D1/D2 sequences of the large (26S) ribosomal RNA gene, and the 5.8S rDNA sequences of $C$. parapsilosis isolates from all three groups were $100 \%$ identical. Only minor differences were observed in ITS2 sequences among the three groups, and considerable dissimilarities were found in the ITS1 sequences.

Candida etchellsii from honey and strawberry jam were in a cluster with $C$. versatilis, C. zemplinina, C. stellata, $C$. cellae (Fig 2). This cluster was an assemblage of phylogenetically diverse species with large interspecific divergences. Other yeasts used in the phylogenetic analysis were isolated from various sources, flowers and pollen nectar, wine, and grapes musk (Table 1). Many of these species are associated with insects, specifically bees, bumblebees and leaf-cutter bees, and many have been reported as the causative agent of spoilage of sugary foods, such as fruit juices and concentrates.

Many of the yeasts related to $C$. parapsilosis group have been implicated in the spoilage of foods, particularly sugary, low- $a_{w}$ foods. Spoilage of high sugar commodities has been reported. Candida davenportii and C. parapsilosis caused fruit juice and soft drink spoilage, $C$. bombicola had been found in concentrated juice and $C$. lactis-condensii had been found in condensed milk as reported by Stratford et al. (2002). Spoilage by $C$. lactis-condensi was relatively uncommon and it is considered to be an opportunist spoilage yeast.

Candida stellata and C. magnoliae are spoilage yeasts in fruit juice concentrates and tomato sauce (Deak and Beuchat 1993). Candida riodocensis and C. cellae have been isolated from pollen-nectar and bees. Candida parapsilosis (Acc GM620527) had been used as fermenting yeast for xylitol production (Erisema et al. 2006). Among the less frequently encountered species, $C$. orthopsilosis had been isolated from fermented cocoa beans (Daniel et al. 2009). Ana et al. (2003) have isolated a new novel species from honey, pollen bee and nectar. The sequence of the D1/D2 domains of the large-subunit rDNA showed that this novel species belongs to the Starmerella cluster, and was most closely related to C. etchellsii. 
Candida parapsilosis, C. metapsilosis, and $C$. orthopsilosis strains collectively formed a distinct lineage which, apart from displaying a loose association with the $C$. parapsilosis group, showed little phylogenetic affinity to any other species examined (Fig 2). Kurtzman and Robnett (1998) observed that strains showing greater than $1 \%$ difference in the ITS region were usually different species, whereas strains with zero to three nucleotide differences were either conspecific or sister species. Alignment of contiguous yeast sequences demonstrated that both singlenucleotide differences and short lengths of sequence diversity due to insertions or deletions existed in the ITS regions among the Candida species. A sequence similarity of $99-100 \%$ exists among C. parapsilosis, C.metapsilosis, C.orthopsilosis, and C. etchellsii.

The genus Candida was the largest in the number of species of the yeast genera and was present in almost every environment. Yeasts of this genus are abundantly distributed in nature. This genus is distributed across the ascomycetous yeast domain, overlapping with other genera according to phylogenetic analysis using ribosomal genes (Kurtzman and Robnett 1998). C. parapsilosis, C. metapsilosis, $C$. orthopsilosis strains collectively form a distinct lineage which apart from displaying a loose association with $C$. parapsilosis group, showed little phylogenetic affinity to any other of the species examined. The sixty-seven isolates could be divided into the $C$. parapsilosis group and the nonC. parapsilosis group, with the exception of one isolate which was clustered on a separate branch.

From all of data obtained in this study, we propose that Indonesian high-sugar foods contain a lot of osmophilic yeasts. Results of this analysis also indicated that most of the isolates belonged to the same clade as the isolates identified in the previous research such as isolates from miso (Suezawa et al. 2006), pollen and flower (Sjamsuridzal et al. 2010), nectar (Matthias 2004), bumblebee (Soon et al. 2003; Michael 2004), grape musk (Belinda et al. 2008), the air (Cristina et al. 2006), seawater (Tekolo et al. 2010). It is suggested that the existence of osmophilic yeasts diversity in products and manufacturing environment needs further investigation. Such knowledge will aid the development of control strategies for food spoilage.

\section{ACKNOWLEDGEMENTS}

We offer our regards and blessings to the Department of Biology, Faculty of Mathematics and Natural Sciences, University of Indonesia that supported us in all respects during the completion of the molecular identification and nucleic acid sequencing.

\section{REFERENCES}

Ali AA, Randall MF. 2002. Implementation of HACCP to bulk condensed milk production line. Food Rev Int 18:177-90.

Ana CPT, Marjorie MM, Nicoli JR, Yasmine A, Rogerio PM, Lachance MA, Rosa CA. 2003. Starmerella meliponinorum sp. nov., a novel ascomycetous yeast species associated with stingless bees. Int J Syst Evol Microbiol 53:339-43.
Ancasi EG, Carrillo L, Benitez-Ahrendts MR. 2006. Moulds and yeasts in bottled water and soft drinks. Rev Argent Microbiol 38:93-6.

Andrew MB, Linton CJ, Oliver D, Palmer MD, Szekely A, Odds FC, Johnson EM. 2009. Pyrosequencing analysis of 20 nucleotides of ITS2 discriminates Candida parapsilosis, Candida metapsilosis, and Candida orthopsilosis. J Clin Microbiol 47:2307-10.

Arias CR, Burns JK, Friedrich LM, Goodrich RM, Parish ME. 2002. Yeast species associated with orange juice. Evaluation of different identification methods. Appl Environ Microbiol 68:1955-61.

Atschul SF, Thomas LM, Schaffer AA, Zhang J, Zhang Z, Miller W, Lipman DJ. 1997. Gapped BLAST and PSI-BLAST: a new generation of protein database search programs. Nucleic Acids Res 25:3389-402.

Belinda ES, Leigh F, Andrew JM, Eileen SS. 2008. The effect of powdery mildew infection of grape berries on juice and wine composition and on sensory properties of Chardonnay wines. Aust J Grape Wine Res 9:28-39.

Beuchat LR. 1983. Influence of water activity on growth, metabolic activities, and survival of yeasts and molds. J Food Protect 46:135-41.

Cristina R, Cinzia R, Cinzia C. 2006. Influence of packaging on spoilage yeast population in minimally processed orange slices. Int J Food Microbiol 109:146-50.

Daniel HM, Jemmy GV, Nicholas CFT, Paul DV, Luc DV. 2009. Yeast diversity of Ghanaian cocoa bean heap fermentations. FEMS Yeast 9:774-83.

Deak T, Beuchat LR. 1993. Yeasts associated with fruit juice concentrates. J Food Prot 56:777-82.

Erisema SH, Simón TL, Virgilio BG, José R, Manuel AV. 2006. Production of detoxified sorghum straw hydrolysates for fermentative purposes. J Sci Food Agric 86:2579-86.

Fitzgerald DJ, Stratford M, Gasson MJ, Narbad A. 2004. The potential application of vanillin in preventing yeast spoilage of soft drinks and fruit juices. J Food Prot 67:391-95.

Kocsube S, Monika T, Csaba V, Ilona D, Miklos P, Istvan P, Judit S, Janos V. 2007. Occurrence and genetic variability of Candida parapsilosis sensu lato in Hungary. J Med Microbiol 56:190-5.

Kurtzman CP, Robnett CJ. 1998. Identification and phylogeny of nuclear large subunit (26S) ribosomal DNA partial sequencess. Antonie Van Leeuwenhoek 73:331-71.

Matthias S. 2004. Species identification and comparative molecular and physiological analysis of Candida zemplinina and Candida stellata. J Basic Microbiol 44:471-9.

Michael BH. 2004. Ecology of yeasts in plant-bumblebee mutualism in Central Europe. FEMS Microbiol Ecol 50:87-100.

Saitou N, Nei M. 1987. The neighbor-joining method: a new method for reconstructing phylogenetic trees. Mol Biol Evol 4:406-25.

Sjamsuridzal W, Oetari A. 2003. Rapid preparation of fungal and bacterial genomic DNA for PCR. Hayati 10:122-4.

Sjamsuridzal W, Oetari A. Kanti A, Saraswati R, Nakashima C, Widyastuti Y, Katsuhiko A. 2010. Ecological and taxonomical perspective of yeast in Indonesia. Microbiol Indones 4:60-8.

Stratford M, Bond CJ, James SA, Roberts IN, Steels H. 2002. Candida davenportii sp. nov., a potential softdrinks spoilage yeast isolated from a wasp. Int J Syst Evol Microbiol 52:1369-75.

Soon GH, Kyung SB, Michel H, Andreas T, Marc AL. 2003. Candida kunwiensis sp. nov., a yeast associated with flowers and bumblebees. Int J Syst Evol Microbiol 53:367-72.

Suezawa Y, Kimura I, Inoue M, Gohda N, Suzuki M. 2006. Identification and typing of miso and soy sauce fermentation yeasts, Candida etchellsii and C. versatilis, based on sequence analyses of the D1/D2 domain of the 26S ribosomal RNA gene, and the region of ITS1, 5.8S ribosomal RNA gene and ITS2. Biosci Biotechnol Biochem 70:348-54.

Tavanti A, Davidson AD, Neil ARG, Martin CJM, Odds FC. 2005. Candida orthopsilosis and Candida metapsilosis spp. nov. to replace Candida parapsilosis groups II and III. J Clin Microbiol 43:284-92. 
Tekolo OM, Jean M, Alfred B, Bernard AP. 2010. The osmotic stress tolerance of basidiomycetous yeasts. FEMS Yeast Res 10:482-91.

Warth AD. 1985. Resistance of yeast spesies to benzoic and sorbic acid and to sulfur dioxide. J Food Prot 48:564-9.
White TJ, Burns T, Lee S, Taylor J. 1990. Amplification and direct sequencing of fungal ribosomal RNA genes for phylogenetics. In: Innis MA, Gelfand DH, Sninsky JJ, White TJ, editors. PCR protocols: a guide to methods and applications. San Diego: Academic Pr. p 315-22. 